\title{
Management of chronic patients during the COVID-19 pandemic: the experience of a referral center for rare hematological disorders in the hardest-hit region in Italy
}

\author{
Irene Motta ${ }^{1,2}$ (D) Alessia Marcon ${ }^{1,2} \cdot$ Maria Domenica Carrabba $^{1} \cdot$ Elena Cassinerio ${ }^{1} \cdot$ Margherita Migone De Amicis $^{1}$. \\ Adriana Branchi ${ }^{1,2} \cdot$ Marianna Giuditta $^{3}$. Diletta Maira ${ }^{1} \cdot$ Giovanna Fabio $^{1} \cdot$ Giovanna Graziadei $^{1}$ • Marina Baldini ${ }^{1}$
}

Received: 4 January 2021 / Accepted: 26 January 2021 / Published online: 2 February 2021

(C) The Author(s), under exclusive licence to Springer-Verlag GmbH, DE part of Springer Nature 2021

Dear Editor,

As the second surge of COVID-19 is reviving the pressure on hospitals, key learnings from the first wave should be of particular value in managing patients suffering from chronic disorders, including rare diseases. Notably, the management of rare diseases is complex, it requires highly specialized health care personnel, and it deals with life-saving non-deferrable therapies. More than 1800 adult patients affected by thalassemia, sickle cell disease (SCD), iron metabolism disorders, porphyria, lysosomal storage disorder, primary immunodeficiencies (PID), and autoinflammatory disorders are followed at the Rare Diseases Center of Fondazione IRCCS $\mathrm{Ca}$ ' Granda Ospedale Policlinico, Milan, Italy.

The outbreak of the COVID-19 pandemic in Italy began on February 20, 2020, and the rapidly evolving situation left a short time to manage the increasing need for beds $[1,2]$. Our hospital reorganization dramatically affected the Rare Diseases Center, which was relocated to make space for intensive care unit beds, with part of the staff reassigned to COVID-19 units.

Patients were encouraged to notify any symptom or concern, and nurses organized a phone call triage (Fig. 1). The systematic use of nasopharyngeal swabs was not suitable

Giovanna Graziadei and Marina Baldini contributed equally to this work.

Irene Motta

irene.motta@unimi.it

1 Rare Diseases Center, General Medicine Unit, Fondazione IRCCS Ca' Granda Ospedale Maggiore Policlinico, Milan, Italy

2 Department of Clinical Sciences and Community Health, Università degli Studi di Milano, Milan, Italy

3 Cardiovascular Disease Unit, Fondazione IRCCS Ca' Granda Ospedale Maggiore Policlinico, Milan, Italy because of the overwhelmed laboratories and the shortage of kits. Thus, only symptomatic cases were tested (Fig. 1). Those who tested positive and did not require hospital admission received their specific therapy in a dedicated COVID-19 area managed by the physicians of the Center, ensuring personalized treatment even during the pandemic [3].

From March to May 2020, scheduled but deferrable consultations were canceled, as well as follow-up exams; the ongoing clinical trials and the psychological service were suspended.

Blood transfusions were guaranteed for more than 200 thalassemia and SCD transfusion-dependent patients, as well as replacement therapy for PID patients. Since intravenous immunoglobulin (IVIG) cannot be administered at home in Italy, some patients shifted from IVIG to subcutaneous immunoglobulin home treatment after training by the nurses of the Center. Since traveling across regions was prohibited, patients from other regions were temporarily transfused/infused in local hospitals (Fig. 1). Home treatment monitoring and issues were managed by phone or e-mail, unless hospital evaluation was required. The vast majority of patients appreciated the possibility of obtaining medical aid while minimizing hospital access and physical contact.

Our experience shows that improving patients' information from physicians and specialized nurses is essential. This approach allowed the continuity of care and the delivery of lifesaving therapies. The active participation of our patients and their support were pivotal.

We are now facing a new wave of COVID 19, and key-learning points from the first wave should be of particular value:

- Remote contact, and testing with nasopharyngeal swab in symptomatic subjects before accessing the hospital for treatment; 


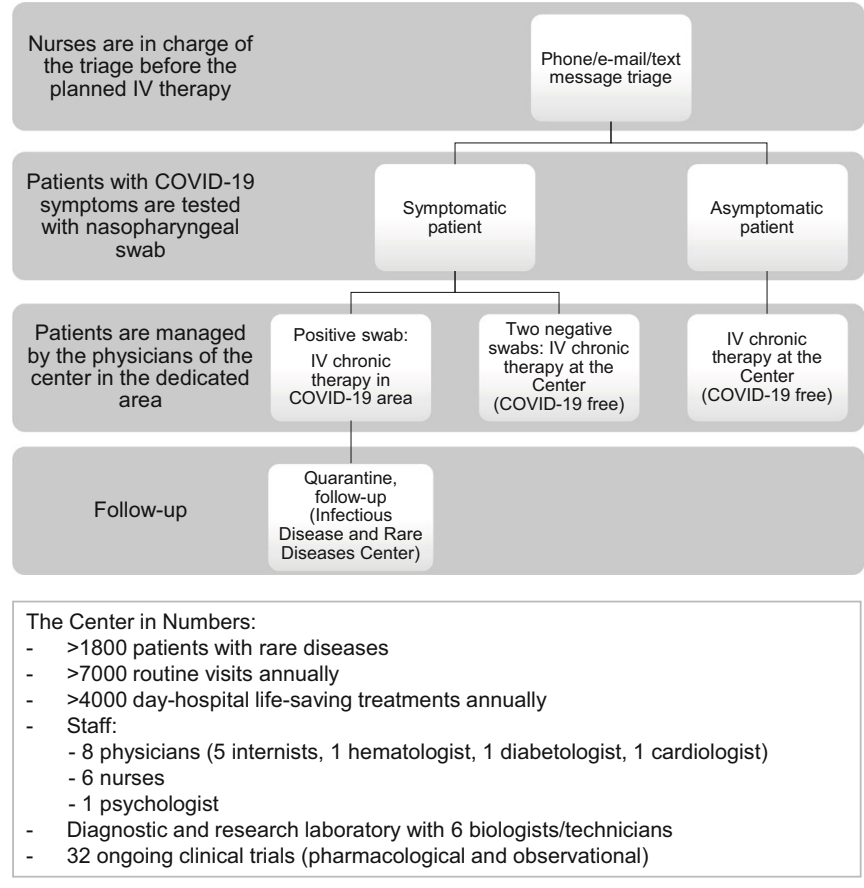

Fig. 1 Impact of the COVID-19 pandemic on treatment management during the first wave (March-May 2020) and second wave (September-December). Treatments were overall regularly administered. Hereby, we report all the TDT, GD1, and AIDs followed in our center and their respective treatments. Moreover, we report only the SCD, S/THAL, Fabry disease, CVID, and XLA which require regular transfusional/ infusional therapy $(*)$. Few patients missed the therapy, mainly by choice. One patient with Fabry disease switched from IV enzyme replacement therapy to oral chaperon treatment $\left({ }^{\S}\right)$. Noteworthy, despite the pandemic, new diagnosis have been made and new treatments have started $(\#)$.

\begin{tabular}{|c|c|c|c|}
\hline & pre-COVID-19 & $\begin{array}{l}\text { CoVID-19 } \\
\text { First wave }\end{array}$ & $\begin{array}{l}\text { CoVID-19 } \\
\text { Second wave }\end{array}$ \\
\hline \multicolumn{4}{|l|}{ TDT } \\
\hline Transfused at the Center & 196 & 181 & $189^{\#}$ \\
\hline Transfused in another center & NA & 15 & 10 \\
\hline Missed treatment & NA & 0 & 0 \\
\hline SARS-CoV-2 positive swab & NA & 6 & 12 \\
\hline \multicolumn{4}{|l|}{ SCD and S/THAL } \\
\hline transfused/exchanged ${ }^{*}$ & 16 & 10 & 10 \\
\hline Transfused at the Center & NA & 0 & 0 \\
\hline Transfused in another center & NA & 6 & 6 \\
\hline Missed treatment & NA & 0 & 0 \\
\hline \\
\hline $\begin{array}{l}\text { GD1 } \\
\text { ERT infused at the Center }\end{array}$ & & & \\
\hline ERT infused in another center & 1 & 0 & 0 \\
\hline ERT Home treatment & 6 & 16 & 16 \\
\hline $\begin{array}{l}\text { Oral treatment } \\
\text { ontion }\end{array}$ & 6 & 6 & $7^{\#}$ \\
\hline Missed treatment & NA & 2 & 2 \\
\hline SARS-CoV-2 positive swab & NA & 0 & 2 \\
\hline \multicolumn{4}{|l|}{ Fabry disease $^{*}$} \\
\hline ERT infused at the Center & 2 & 0 & 0 \\
\hline ERT Home treatment & 5 & 5 & 5 \\
\hline Oral treatment & 4 & $5^{5}$ & $6^{\#}$ \\
\hline Missed treatment & NA & 1 & 1 \\
\hline SARS-CoV-2 positive swab & NA & 1 & 0 \\
\hline \multicolumn{4}{|l|}{ CVID and XLA* } \\
\hline Infused at the Center & 39 & $28^{\#}$ & $23^{\#}$ \\
\hline Infused in another center & 4 & 6 & 2 \\
\hline Home treatment (SCIG) & 22 & $31^{\#}$ & $42^{\#}$ \\
\hline Missed treatment & NA & 3 & 0 \\
\hline SARS-CoV-2 positive swab & NA & 0 & 9 \\
\hline \multicolumn{4}{|l|}{ AlDs } \\
\hline Infused at the Center & 4 & $3^{\#}$ & 2 \\
\hline Home treatment & 0 & 0 & \\
\hline Oral treatment & 65 & 68 & $68^{\#}$ \\
\hline Missed treatment & NA & 1 & 0 \\
\hline SARS-CoV-2 positive swab & NA & 0 & 0 \\
\hline
\end{tabular}

During the second wave, a higher number of patients had a symptomatic form of SARS-Cov-2 infection compared to the first wave, consistent with the general population epidemiology. IV, intravenous; COVID-19, coronavirus disease; TDT, transfusion-dependent thalassemia; SCD, sickle cell disease; S/THAL, sickle/thalassemia; GD1, Gaucher disease type 1; ERT, enzyme replacement therapy; CVID, common variable immunodeficiency; XLA, X-linked agammaglobulinemia; AIDs, autoinflammatory diseases; SCIG, subcutaneous immunoglobulin; NA, not applicable
- Dedicated COVID+ areas for life-saving treatments to ensure individualized therapy;

- Home delivery and monitoring of oral and IV drugs to avoid unnecessary hospital access;

- The continuation of clinical trials, ensuring the safety of enrolled patients;

- Psychological support for chronic fragile patients.

The COVID-19 pandemic is a unique, intense, personal, and professional emotional experience. The resilience of health care professionals demonstrated that we can reach our full human potential to care for rare, complex, and fragile patients. Institutions should ensure dedicated and specialized personnel, areas, and time for chronic patients' care.

Acknowledgements We thank all the nurses and the administrator of the Center, whose collaboration and energy are inspiring: Simona Carboni, Damiana Bellotta, Katia Bussoli, Simona Orlando, Paolo Paludetti,
Roberto Saiani, Maria Tabini, and Marina Zarantonello. We thank all the patients whose support was essential. This work is generated within the European Reference Network on Rare Hematological Diseases (ERNEuroBloodNet). FPA 739541.

Author contributions IM wrote the paper. AM, MDC, and MMDA collected the data. All authors take care of patients and contributed to critical revision and final approval of the version to be published.

Declarations All procedures performed in studies involving human participants were in accordance with the ethical standards of the institutional and/or national research committee and with the 1964 Helsinki declaration and its later amendments or comparable ethical standards. This is an observational study. The Research Ethics Committee "Comitato Etico Milano Area 2 " has confirmed that no ethical approval is required.

Conflict of interest IM received lecture honoraria from SanofiGenzyme and is member of Sanofi-Genzyme and Amicus Therapeutics advisory board. AM, MDC, EC, MMDA, AB, MG, DM, GF, GG, MB declare that they have no conflict of interest. 


\section{References}

1. Grasselli G, Pesenti A, Cecconi M (2020) Critical care utilization for the COVID-19 outbreak in Lombardy, Italy: early experience and forecast during an emergency response. JAMA. 323(16):1545-1546

2. Auxilia F, Maraschini A, Bono P et al (2020) COVID-19: new scenario old problems. Acta Biomed 91(Suplement9):90-91. https:// doi.org/10.23750/abm.v91i2-S.10119
3. Motta I, Migone De Amicis M, Pinto VM et al (2020) SARS-CoV-2 infection in beta thalassemia: preliminary data from the Italian experience. Am J Hematol 95(8):E198-E199. https://doi.org/10.1002/ ajh.25840.

Publisher's note Springer Nature remains neutral with regard to jurisdictional claims in published maps and institutional affiliations. 